\title{
Everything You Always Wanted to Know About Cone Beam Computed Tomography: 3D-Roentgen.ch
}

\section{Ozdoba}

Received: 28 June 2013 / Published online: 18 July 2013

C) Springer-Verlag Berlin Heidelberg 2013

Cone Beam Computed Tomography (CBCT; in German "Digitale Volumentomographie"; digital volume tomography) has become a standard tool in ear, nose, and throat (ENT) [1], maxillofacial [2], and dental X-ray in recent years [3]. Although its merits and potential are sometimes overestimated $[4,5]$, radiology departments increasingly add such a device to their equipment pool to satisfy their clientele's demands.

The website that I present in this issue is based in Switzerland; the text is in German only (Fig. 1), but the tabular overviews should be understandable for the English speaking readers as well.

The site is run by a company that specializes in IT tools and equipment for dental surgery; the information is an independent collection of information gathered from the various manufacturers.

"3D-Roentgen" lists technical data (Fig. 2) for all CBCT machines currently available in Switzerland. Furthermore, the data sheets/brochures for all these systems are available by direct links to the manufacturers' websites.

I was positively astonished to see that, although the site is maintained by a commercial company, it is completely free of any advertising. "3D-Roentgen" provides the data but does neither display banners or any other kind of advertising nor does it give recommendations for specific devices.

Therefore, I can recommend this site as a good overview if you are interested in getting a CBCT machine for your practice or clinical department.

All screenshots are from www.3d-roentgen.ch (taken in June 2013).

C. Ozdoba $(\bowtie)$

Bern, Switzerland

e-mail: christoph.ozdoba@insel.ch

\section{References}

1. Hodez C, Griffaton-Taillandier C, Bensimon I. Cone-beam imaging: applications in ENT. Eur Ann Otorhinolaryngol Head Neck Dis. 2011;128:65-78.

2. Krishnamoorthy B, Mamatha N, Kumar VA. TMJ imaging by CBCT: current scenario. Ann Maxillofac Surg. 2013;3:80-3.

3. Scarfe WC, Farman AG, Sukovic P. Clinical applications of conebeam computed tomography in dental practice. J Can Dent Assoc. 2006;72:75-80.

4. Secrétariat scientifique, Office fédéral de la santé publique: Stellungnahme der Eidgenössischen Kommission für Strahlenschutz und Überwachung der Radioaktivität zur Digitalen Volumentomographie. Berne, Juillet 2010.

5. Horner K. Cone-beam computed tomography: time for an evidence-based approach. Prim Dent J. 2013;2:22-31. 
Fig. 1 Digital volume tomography website

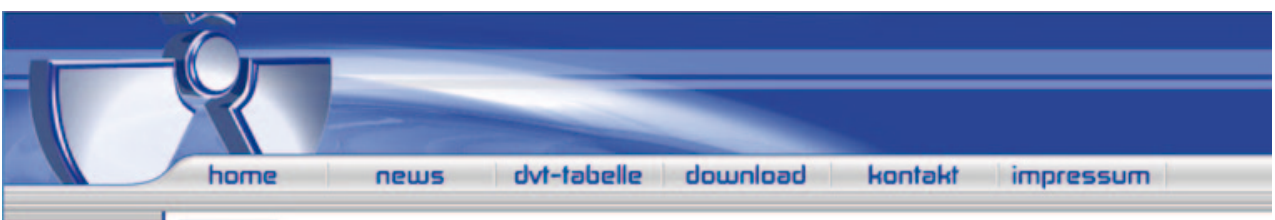

Mehr Infos

DVT-Gerbite:

GXCB 500

Newtom 5G

Kavo 3D exam

Kodak 9000 3D

Kodak9500

Sirona Galilieos

Orthophos XG3D

Pax Uni 3D

Paxzenith3D

Sensoren.

Technologien

rescos

Info:

Hier

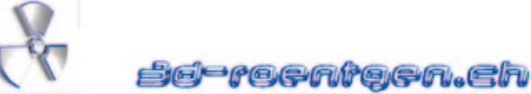

Digitale Volumentomographie
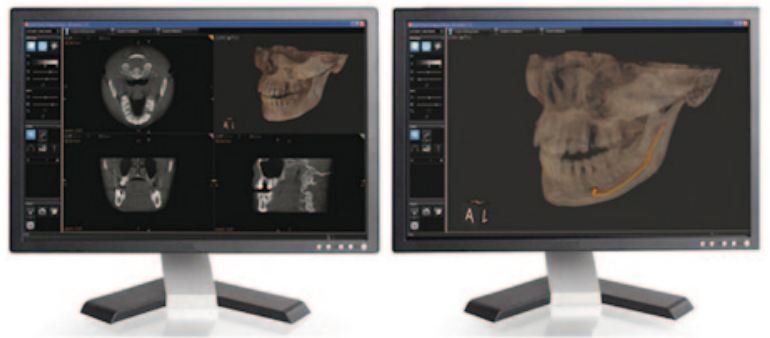

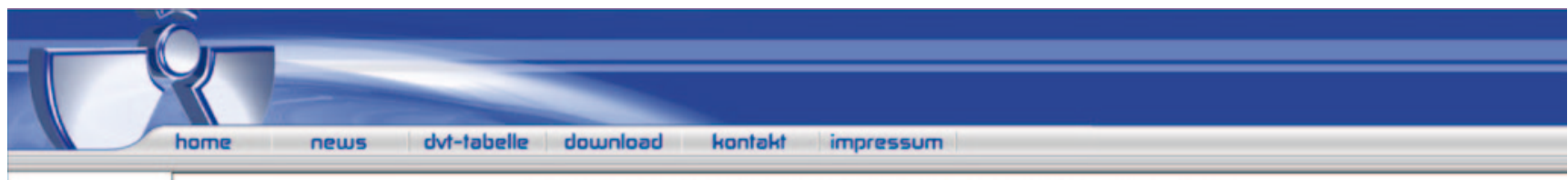

In der Schweiz angebotene Digitale Volumentomographen (sortiert nach Fov)

- Klick auf Typ = Prospekt

- Klick auf das Mass des Mindestraumbedarfs = geratespezifische Massskizze

- Klick auf Detektor = Informationen zur entsprechenden Deteiktortechnologie

- Die Dosiswerte entsprechen nicht einem standardisierten, vergleichbaren Messwert

- Alle Daten beruhen auf Angaben der Hersteller oder deren Schweizer Vertretung

\begin{tabular}{|c|c|c|c|c|c|c|}
\hline $\begin{array}{l}\text { DVT } \\
\text { Features-Übersicht }\end{array}$ & & $=$ & & $4 \sqrt{2}$ & $\sqrt{2}$ & $I^{m}$ \\
\hline & $\begin{array}{l}\text { Carestream } \\
900030 \\
\end{array}$ & Pax Uni 30 & $\begin{array}{l}\text { Planmeca } \\
\text { Promax 3Ds }\end{array}$ & $\begin{array}{l}\text { Kavo } \\
\text { Pan eXam Plus }\end{array}$ & $\begin{array}{l}\text { Soredex } \\
\text { Cranex } 30 \\
\end{array}$ & $\begin{array}{l}\text { Sirona } \\
\text { Orthophos XG 30 }\end{array}$ \\
\hline Hersteller & $\begin{array}{l}\text { Kodak } \\
\text { (Trophy, F) }\end{array}$ & $\begin{array}{l}\text { VATECH Co, LAd S E-Woo, } \\
\text { Sudkores }\end{array}$ & Planmeca. Fi & DaloDEx Group Ok, $F$ & Sotedex or. Fi & Sirona. D \\
\hline Richtpreis & C 54.999.- & $a b<>4900$ & $a b(81217=$ & $a b<85000$. & $a b<70$ 's50. & $a b<88^{\circ} 900$. \\
\hline $\begin{array}{l}\text { Mindestraumbedarf } \\
(T \times B \times A+)\end{array}$ & $1.7 \times 1.5 \times 2.25 \mathrm{~m}$ & $1.6 \times 1.2 \times 2.35 \mathrm{~m}$ & $1.5 \times 1.63 \times 2.43 \mathrm{~m}$ & $1.5 \times 1.1 \times 2.05(2.45) \mathrm{m}$ & $1.5 \times 1.2 \times 2.15(2.45) \mathrm{m}$ & $1.5 \times 1.2 \times 2.25 \mathrm{~m}$ \\
\hline Patientenpositionierung & sitzend / stehend & sitzend/stehend & sitzend/stehend & sitzend / stehend & sitzend / stehend & sitzend / stehend \\
\hline Deteltor & CMOS Flatpanel & CMOS Flatpanel & $\begin{array}{l}\text { Amorpher Silizium } \\
\text { Flachdetekter }\end{array}$ & CMOS Fiatpanel & CMOS Flatpanel & CMOS Flatpane! \\
\hline $\begin{array}{l}\text { Aufnahmevolumen } \\
\text { FOV }(d \times h)\end{array}$ & $\begin{array}{l}5 \times 3.7 \mathrm{~cm} \\
\text { Stiching: } 7.5 \times 3.7 \mathrm{~cm}^{*}\end{array}$ & $\begin{array}{c}5 \times 5 \mathrm{~cm} \\
(8 \times 5 \mathrm{~cm})^{*} \\
(12 \times 8.5 \mathrm{~cm})^{*}\end{array}$ & $\begin{array}{c}5 \times 5 \mathrm{~cm} \\
8 \times 5 \mathrm{~cm} \\
\text { Stiching: } 10 \times 11 \mathrm{~cm}\end{array}$ & $\begin{array}{l}4.1 \times 6.1 \mathrm{~cm} \\
7.8 \times 6.1 \mathrm{~cm}\end{array}$ & $\begin{array}{l}4.1 \times 6.1 \mathrm{~cm} \\
\text { Optionals } 7.8 \times 6.1 \mathrm{~cm}\end{array}$ & $\begin{array}{l}5 \times 5.5 \mathrm{~cm} \\
8 \times 8 \mathrm{~cm}\end{array}$ \\
\hline Auflosung & $\begin{array}{c}0.076 \mathrm{~mm} \\
\text { Stiching: } 0.2 \mathrm{~mm}\end{array}$ & $0.186 \mathrm{~mm}$ & $0.1 \mathrm{bzw}, 0.2 \mathrm{~mm}$ & $0.133 \mathrm{bzw}, 0.25 \mathrm{~mm}$ & $0.133 \mathrm{bzw}, 0.2 \mathrm{~mm}$ & $0.1 \mathrm{bzw} .0 .16 \mathrm{~mm}$ \\
\hline Aufnahmezeit & $14 \mathrm{Sec}$ & $8.3 \cdot 20 \mathrm{sec}$ & $18 \mathrm{Sec}$ & $10-20 \mathrm{Sec}$ & $10-20 \mathrm{Sec}$ & $14 \mathrm{Sec}$ \\
\hline Rekonstruktionsdaver & $<40 \mathrm{Sec}$ & $<40$ Ses & $30 \cdot 150 \mathrm{Sec}$ & $2-3$ Min & $<120 \mathrm{Sec}$ & $<2$ Min \\
\hline $\begin{array}{l}\text { Patientendosis } \\
\text { (Herstellerangaben) }\end{array}$ & $\begin{array}{c}11-19 \mu \mathrm{Sv} \\
\text { Stiching: } 31.3 \mathrm{uSv}\end{array}$ & Ca. $30 \mu \mathrm{Sv}$ & $18-<200 \mu \mathrm{Sv}$ & $39-126 \mu \mathrm{Sv}$ & & $\begin{array}{l}\text { ICRP 2007: 43-175 uSv } \\
\text { (Standard: } 100 \text { uSv) }\end{array}$ \\
\hline Rohrenspannung (kV) & $60-90$ & $40 \cdot 90$ & $54-84$ & $57 \cdot 90$ & $57 \cdot 90$ & $60 \cdot 90$ \\
\hline Rohrenstrom $(\mathrm{mA})$ & 2.15 & $2 \cdot 10$ & $1 \cdot 16$ & $4 \cdot 16$ & $4 \cdot 16$ & $3-16$ \\
\hline Brennfleck $(\mathrm{mm})$ & $0.5 \times 0.5$ & $0.35 \times 0.5$ & $0.5 \times 0.5$ & $0.5 \times 0.5$ & $0.5 \times 0.5$ & $0.5 \times 0.5$ \\
\hline Rotationswinkel & $360^{\circ}$ & $220^{\circ}$ & $200^{\circ}$ & $2 \times 180^{\circ}$ assymetrisch & & \\
\hline $\begin{array}{l}\text { Anzahl Projelctionen } \\
\text { wahrend des Umlauts }\end{array}$ & 360 & $\min .440 / \max .715$ & 300 & $234 / 486 / 608 / 1260$ & $234 / 486 / 608 / 1260$ & $200 / 500$ \\
\hline
\end{tabular}

Fig. 2 Features of cone beam computed tomography machines currently available in Switzerland 